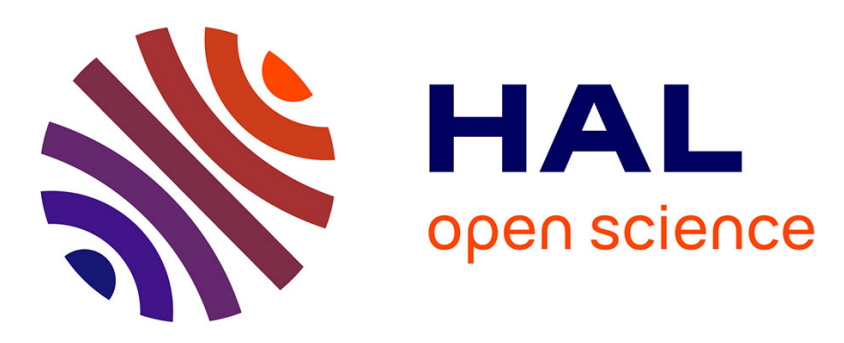

\title{
The anti-diabetic drug metformin suppresses self-renewal and proliferation of trastuzumab-resistant tumor-initiating breast cancer stem cells
}

Alejandro Vazquez-Martin, Cristina Oliveras-Ferraros, Sonia Del Barco, Begoña Martin-Castillo, Javier A. Menendez

\section{To cite this version:}

Alejandro Vazquez-Martin, Cristina Oliveras-Ferraros, Sonia Del Barco, Begoña Martin-Castillo, Javier A. Menendez. The anti-diabetic drug metformin suppresses self-renewal and proliferation of trastuzumab-resistant tumor-initiating breast cancer stem cells. Breast Cancer Research and Treatment, 2010, 126 (2), pp.355-364. 10.1007/s10549-010-0924-x . hal-00583557

\section{HAL Id: hal-00583557 \\ https://hal.science/hal-00583557}

Submitted on 6 Apr 2011

HAL is a multi-disciplinary open access archive for the deposit and dissemination of scientific research documents, whether they are published or not. The documents may come from teaching and research institutions in France or abroad, or from public or private research centers.
L'archive ouverte pluridisciplinaire HAL, est destinée au dépôt et à la diffusion de documents scientifiques de niveau recherche, publiés ou non, émanant des établissements d'enseignement et de recherche français ou étrangers, des laboratoires publics ou privés. 


\title{
Breast Cancer Research and Treatment
}

\section{The anti-diabetic drug metformin suppresses Self-renewal and proliferation of trastuzumab-resistant Tumor-initiating breast cancer stem cells}

\author{
Alejandro Vazquez-Martin 1,2 a \\ Cristina Oliveras-Ferraros ${ }^{1,2}$ a \\ Sonia Del Barco 1,2 \\ Begoña Martin-Castillo 1,2 \\ Javier A. Menendez 1,2 * \\ ${ }^{1}$ Catalan Institute of Oncology (ICO) \\ ${ }^{2}$ Girona Biomedical Research Institute (IdIBGi) \\ Girona, Catalonia, SPAIN
}

a Both authors contributed equally to this research and are listed in random order

*Address for correspondence:

JAVIER A. MENENDEZ, Ph.D.

Catalan Institute of Oncology, Girona (ICO-Girona)

Dr. Josep Trueta University Hospital

Ctra. França s/n, E-17007 Girona, Catalonia (Spain)

Phone: + 34-972-225-834 Ext. 2553

Fax: + 34-972-217 344

E-mail:jmenendez@iconcologia.net

Running head: Metformin and tumor-initiating cells in HER2 overexpressors Key words: Metformin; Trastuzumab; HER2; Stem Cells; Tumor-initiating cells 


\section{Summary}

We here demonstrate that the anti-diabetic drug metformin interacts synergistically with the anti-HER2 monoclonal antibody trastuzumab (Tzb; Herceptin ${ }^{\mathrm{TM}}$ ) to eliminate stem/progenitor cell populations in HER2-gene amplified breast carcinoma cells. When using the mammosphere culture technique, graded concentrations of singleagent metformin (range 50-1000 $\mu \mathrm{mol} / \mathrm{L}$ ) were found to dose-dependently reduce the number of mammospheres formed by SKBR3 (a Tzb-naïve model), SKBR3 TzbR (a model of acquired auto-resistance to Tzb) and JIMT-1 (a model of refractoriness to Tzb and other HER2-targeted therapies ab initio) HER2-overexpressing breast cancer cells. Single-agent Tzb likewise reduced mammosphere-forming efficiency (MSFE) in Tzbnaïve SKBR3 cells but it failed to significantly decrease MSFE in Tzb-resistant SKBR3 TzbR and JIMT-1 cells. Of note, CD44-overexpressing Tzb-refractory SKBR3 TzbR and JIMT-1 cells retained an exquisite sensitivity to single-agent metformin. Concurrent combination of metformin with Tzb synergistically reduced MSFE as well as the size of mammospheres in Tzb-refractory $\mathrm{SKBR}_{3}$ TzbR and JIMT-1 cells. Flow cytometry analyses confirmed that metformin and Tzb functioned synergistically to down-regulate the percentage of Tzb-refractory JIMT-1 cells displaying the $\mathrm{CD} 44^{\text {pos }} \mathrm{CD} 24^{\text {neg/low }}$ stem/progenitor immunophenotype. Given that MSFE and mammosphere size are indicators of stem self-renewal and progenitor cell proliferation, respectively, our current findings reveal for the first time that: a.) Tzb refractoriness in HER2 overexpressors can be explained in terms of Tzb-resistant/CD44overexpressing/tumor-initiating stem cells; b.) metformin synergistically interacts with Tzb to suppress self-renewal and proliferation of cancer stem/progenitor cells in HER2-positive carcinomas. 


\section{Introduction}

For decades, breast cancer (BC) initiation and progression has been regarded as a multistep process that is reflected by the progressive genetic alterations that drive the transformation of normal human cells into highly malignant derivatives [1]. In recent years, the experimental demonstration of tumor-initiating cells (popularly known as cancer stem cells) rather supports tumor hierarchy as a fundamental concept in the biology of $\mathrm{BC}$ disease [2]. According to the cancer stem cell hypothesis, primary BC contain subpopulations of replenishing stem-like cells (i.e. tumor-initiating cells) with the ability of both self-renew and give rise to phenotypically diverse progeny [3]. In other words, solely the tumor-initiating cells have the ability to proliferate extensively, give rise to differentiated cells and form new tumors. When using cytotoxics or radiation, the bulk $\mathrm{BC}$ cell population decreases, but the proportion of tumor-initiating cells is higher than before treatment, thus indicating that conventional therapeutics efficiently kill differentiated BC cells whereas BC stem cells -by their nature- are intrinsically refractory thereby allowing $\mathrm{BC}$ regrowth $[4,5]$. The fact that tumorinitiating cells display intrinsic resistance to many conventional treatments might therefore explain the current limitations of these therapeutic approaches in curing breast BC.

Although many novel molecularly targeted agents (e.g. monoclonal antibodies, tyrosine kinase inhibitors [TKIs], etc.) have been also designed to target rapidly proliferating $\mathrm{BC}$ cancer cells -so many tumor-initiating cells might also be relatively insensitive to these agents- we are beginning to accumulate evidence that anti-HER2 therapeutics can effectively target tumor-initiating cells derived from HER2overexpressing BC $[5,6]$. In analogy with genes such as Notch, Hedgehog, Wnt and ras, initially identified as oncogenes but shown subsequently to be actively involved in selfrenewal and survival of tumor-initiating cells, HER2 may also constitute a pivotal 
molecule in BC stem cells. The notion that HER2 signaling can drive tumor-initiating cells in certain HER2-positive BC is supported by the following findings: 1.) HER2 overexpression in multiple $\mathrm{BC}$ cell lines significantly increases the fraction of cells positive for ALDH1 (aldehyde dehydrogenase) [7] -a marker of normal and cancerous human mammary epithelial cells with stem/progenitor properties- and enhances the tumor-initiating component that drives tumorigenesis, invasion and metastasis [8]; 2.) Pioneering findings by $\mathrm{Li}$ et al demonstrated that, conversely to chemotherapy treatment, the epidermal growth factor receptor (EGFR)/HER2 dual TKI lapatinib, decreases the subpopulation of chemotherapy-resistant BC initiating cells (measured by calculating the percentage of cells with the $\mathrm{CD} 44^{\mathrm{pos}} / \mathrm{CD} 24^{\text {neg/low }}$ stem/progenitor immunophenotype and the ability to form mammospheres in vivo as an indication of self-renewal) [5], and; 3.) Tumor-initiating cells of HER2-positive BC have been shown to express the highest HER2 levels and been sensitive to the anti-HER2 monoclonal antibody trastuzumab [Tzb; Herceptin ${ }^{\mathrm{TM}}$ ] [6]. Intriguingly, recent observations have suggested also that clinical resistance to Tzb and other HER2-targeted therapies may be driven by stem-like BC cells within HER2-positive BC. Indeed, a variety of possible mechanisms of escape from Tzb involves many of the same markers that have been implicated in the biology of cancer stem cells (e.g. loss or blockade of the Tzb binding site at the extracellular domain of HER2 upon overexpression of the stem cell marker CD44, signaling through alternative receptor pathways via the stem cell markers CXCR4 and $\beta 1$ integrin, activation of Notch signaling to promote Tzb resistance via enhanced self-renewal/proliferation of progenitor cells or induction of epithelia-tomesenchymal transition, etc.) [Reviewed in 9]. Therefore, a major challenge now is: 1.) To define the sensitivity of the sub-population of tumor-initiating (BC stem) cells to current and novel anti-HER2 BC therapies; and 2.) To discover agents and strategies 
that can target HER2-positive $\mathrm{BC}$ and HER2-positive $\mathrm{BC}$ relapse at their apparent source of tumor-initiating (BC stem) cells.

Culturing BC cells in so-called "mammosphere culture" has been proposed as a reliable method for enriching sub-populations of putative $\mathrm{BC}$ tumor-initiating stem cells [10]. Mammosphere culture could provide a highly appropriate model for studying the sensitivity of the tumor-initiating stem cells within a heterogeneous BC cell population to therapeutic agents. Thus, a comparison of efficacy using mammospheres from BC cells lines or mammospheres cultured from individual pleural effusions as targets would allow evaluation of the consistency of effectiveness of a specific therapy. Taking advantage of the ability of BC stem cells to form multicellular "microtumors" in non-adherent and non-differentiating conditions (i.e. "mammospheres" $[11,12]$ ) and considering novel data showing the unexpected ability of the anti-diabetic drug metformin to preferentially kill BC stem cells [13], we recently decided to explore if metformin may alter self-renewal and proliferation of tumor-initiating stem cells in BC in vitro models representative of Tzb-responsive and Tzb-refractory HER2-gene amplified $\mathrm{BC}$ disease. We have assessed also, at the level of mammosphere-initiating cells, the relevance of well-recognized BC stem cell markers (i.e. CD44) in the phenomena of both acquired and de novo refractoriness to the anti-HER2 monoclonal antibody Tzb. Finally, we have explored the nature of the (anti-BC stem cell) interaction (i.e. antagonism, addition, and synergism) between metformin and Tzb in Tzb-naïve and Tzb-refractory HER2-overexpressing BC models. 


\section{MATERIALS and METHODS}

Materials. Trastuzumab (Herceptin ${ }^{\mathrm{TM}}$ ) was provided by Pharmacy Department, Hospital Universitario de Girona Dr. Josep Trueta (Girona, Spain), solubilized in bacteriostatic water for injection containing 1.1\% benzyl alcohol (stock solution at 21 $\mathrm{mg} / \mathrm{ml})$, stored at $4^{\circ} \mathrm{C}$ and used within one month. Metformin hydrochloride (1,1dimethylbiguanide hydrochloride) was purchased from Sigma Chemical (St. Louis, MO, USA), solubilized in phosphate-buffered saline (PBS; stock solution at $1 \mathrm{~mol} / \mathrm{L}$ ), stored at $-20{ }^{\circ} \mathrm{C}$ and used within two weeks.

Culture Conditions. SKBR3, MDA-MB-468 and MDA-MB-231 human breast cancer cells were obtained from the American Type Culture Collection (ATCC) and they where routinely grown in Improved MEM (IMEM; BioSource International; Invitrogen S.A., Barcelona, Spain) supplemented with 5\% fetal bovine serum (FBS) and $2 \mathrm{mM} \mathrm{L-}$ Glutamine. SKBR3 TzbR cells (POOL1), an in vitro model with acquired autoresistance to Tzb that grows in the continuous presence of $100 \mu \mathrm{g} / \mathrm{mL} \mathrm{Tzb}$, has been developed in our laboratory as previously described [14]. JIMT-1 human breast cancer cell line was established at Tampere University from a ductal carcinoma pleural metastasis of a 62-year-old-BC patient who did not respond to Tzb [15]. JIMT-1 cell line is available from the German Collection of Microorganisms and Cell Cultures (http://www.dsmz.de/). JIMT-1 cells were grown in F-12/DMEM (1:1) supplemented with $10 \% \mathrm{FBS}$ and $2 \mathrm{mM} \mathrm{L}$-glutamine. Cells were maintained at $37^{\circ} \mathrm{C}$ in a humidified atmosphere of $95 \%$ air and $5 \% \mathrm{CO}_{2}$. Cells were screened periodically for Mycoplasma contamination. 
Mammosphere culture. Mammospheres were generated from single cells of SKBR3, SKBR3 TzbR, and JIMT-1 cell lines seeded at $10^{3}$ cells $/ \mathrm{cm}^{2}$ in six-well ultralow attachment plates (Corning Inc.). Sphere medium (F-12/DMEM containing $5 \mathrm{mg} / \mathrm{mL}$ insulin, $0.5 \mathrm{mg} / \mathrm{mL}$ hydrocortisone, 2\% B27 [Invitrogen Ltd.], and $20 \mathrm{ng} / \mathrm{mL}$ epidermal growth factor) was prepared as originally described by Dontu et al. [11].

Mammosphere-forming efficiency (MSFE). MSFE was calculated as the number of spheres-like structures (large diameter $>50 \mu \mathrm{m}$ ) formed in 7 days divided by the original number of cells seeded and expressed as percentage means $( \pm \mathrm{SD})$

\section{Flow cytometry. $C$}

ell cultures growing in regular medium (or supplemented with Tzb and/or metformin as specified), were washed once with phosphate-buffered saline (PBS) and then harvested with $0.05 \%$ trypsin/0.025\% EDTA into single cell suspensions. Detached cells were washed with PBS containing 1\% FBS and 1\% penicillin/streptomycin (wash buffer), counted, and resuspended in the wash buffer $\left(10^{6}\right.$ cells $\left./ 100 \mu l\right)$. Combinations of fluorochrome-conjugated monoclonal antibodies obtained from BD Biosciences (San Diego, CA, USA) against human CD44 (FITC; cat.\#555478) and CD24 (PE; cat.\#555428) or their respective isotype controls were added to the cell suspension at concentrations recommended by the manufacturer and incubated at $4{ }^{\circ} \mathrm{C}$ in the dark for 30-40 min. Labeled cells were washed in the wash buffer to eliminate unbound antibody, then fixed in PBS containing $1 \%$ paraformaldehyde, and then analyzed no longer than $1 \mathrm{~h}$ post-staining on a BD FacScalibur (BD Biosciences). 
Statistical analysis. Results are presented as the mean \pm SD for at least three repeated individual experiments for each group. Analysis was done using XLSTAT 2010 (Addinsoft $^{\mathrm{TM}}$ ). $\mathrm{P} \leq \mathrm{0.01}$ was considered statistically significant. 


\section{RESULTS}

\section{Metformin is as efficient as Tzb at reducing MSFE in Tzb-naïve SKBR3 BC}

cells. The mammosphere culture technique was employed to assess whether Tzb and metformin, as single agents, may significantly reduce MSFE in Tzb-naïve SKBR3 BC cells (Fig. 1A). A clinically relevant concentration of Tzb was sufficient to significantly decrease MSFE in HER2-overexpressing SKBR3 cells ( $\sim 35 \%$ MSFE reduction at 10 $\mu \mathrm{g} / \mathrm{mL}$ Tzb; Fig. 1B). Similarly, a 35\% MSFE reduction was obtained when sphere cultures of SKBR3 cells were carried out in the presence of $100 \mu \mathrm{mol} / \mathrm{L}$ metformin (Fig. 1B). Indeed, a dose-dependent reduction of MSFE was clearly observed when SKBR3 cells were cultured in sphere medium supplemented with graded concentrations of metformin (up to $75 \%$ reduction at $1000 \mu \mathrm{mol} / \mathrm{L}$ metformin; Fig. 1A,B). Remarkably, metformin treatment not only reduced MSFE but caused further morphological changes to SKBR3 mammospheres. Thus, SKBR3 mammospheres formed in the presence of 50 and $100 \mu \mathrm{g} / \mathrm{mL}$ metformin exhibited a significant disintegration of the spherical mammospheres structures observed in untreated SKBR3 control cells (Fig. 1A). When sphere cultures of $\mathrm{SKBR}_{3}$ cells were carried out in the presence of concurrent Tzb and metformin, we observed mostly additive interactions of both agents when analyzing fold-reductions of MSFE in SKBR3 cells (Fig. 1C).

\section{Acquisition of auto-resistance to Tzb can be explained in terms of "Tzb-} resistant" HER2-positive BC stem/progenitor cells. Given the remarkable heterogeneity of BC disease, it has been suggested that the characteristics of HER2positive BC tumor-initiating cells may evolve under the selection pressure of Tzb to drive the emergence of "Tzb-resistant" HER2-positive BC stem/progenitor cells [9]. To evaluate this hypothesis, we took advantage of SKBR3 TzbR cells (POOL1), which were 
obtained in our laboratory by continuously culturing HER2-dependent SKBR3 parental cells in the presence of high-doses of Tzb for more than 10 months [14]. This Tzbconditioned SKBR3 derivative optimally grows in the presence of $100 \mu \mathrm{g} / \mathrm{mL}$ Tzb. Unlike Tzb-sensitive SKBR3 parental cells, which formed discrete and spherical mammospheres, SKBR3 TzbR cells cultured in sphere medium formed larger \& irregular mammosphere-like structures (Fig. 2A). To evaluate whether changes in gross mammosphere structure related to changes in the expression of the stem cell markers involved also in Tzb resistance, we employed flow cytometry to examine cell surface distribution of CD24 and CD44 markers before and after acquisition of Tzb autoresistance. Tzb-naïve SKBR3 parental cells likewise exhibited a significantly enriched $\mathrm{CD} 24^{\mathrm{pos}} / \mathrm{CD} 44^{\text {neg }}(\sim 90 \%)$ cell population, which is consistent with the luminal-type classification of SKBR3 BC cells (Fig. 2A, left). Interestingly, 55\% of the SKBR3 TzbR cell population displayed a CD24pos/CD24 $4^{\text {pos }}$ immunophenotype, which to some extent resembled the $\mathrm{CD} 24 / \mathrm{CD} 44$ distribution that can be observed in highly-metastatic MDA-MB-468 basal-like BC cells (Fig. 2A, right). Moreover, mammosphere-initiating cells within SKBR3 TzbR cell populations did exhibit resistance to Tzb as MSFE remained largely unaltered when SKBR3 TzbR sphere cultures were performed in the presence of Tzb doses as high as $100 \mu \mathrm{g} / \mathrm{mL}$ (Fig. 2B,C).

\section{Metformin interacts synergistically with Tzb at reducing MSFE in HER2-} overexpressing BC cells with acquired resistance to Tzb. In light of these findings, we sought to assess whether metformin could reverse acquired autoresistance to Tzb by targeting "Tzb-resistant” HER2-positive BC stem/progenitor cells. Of note, Tzb-refractory mammosphere-initiating cells were exquisitely sensitive to metformin. $100 \mu \mathrm{mol} / \mathrm{L}$ metformin reduced MSFE by about $50 \%$ while $1000 \mu \mathrm{mol} / \mathrm{L}$ 
metformin completely suppressed mammosphere formation in SKBR3 TzbR cells (Fig. 2A,B). Moreover, synergistic interactions between Tzb and metformin were found throughout the entire range of metformin concentrations (Fig. 2C). Thus, the ability of $500 \mu \mathrm{mol} / \mathrm{L}$ metformin to decrease SKBR3 TzbR MSFE increased from about 5 times when used as single-agent to more than 17 times when combined with Tzb.

\section{Metformin interacts synergistically with Tzb to reduce MSFE in JIMT-1 BC} cells, an in vitro model of refractoriness to HER2-targeted therapies ab

initio. To validate further the notion that metformin may attack Tzb-resistant tumorinitiating stem cells, we took advantage of the de novo Tzb resistance in a cell line (JIMT-1) isolated from the pleural fluid of a HER2-positive BC patient with progressive disease on Tzb [15]. Unlike Tzb-sensitive HER2-overexpressing SKBR3 cells and similarly to SKBR3 TzbR cells with acquired auto-resistance to Tzb, Tzb-refractory JIMT-1 cells cultured in sphere medium for 7 days formed irregular and somewhat loosely-adhered clumps of cells (Fig. 3A). Although treatment with single-agent Tzb reduced both the number and the size of JIMT-1 mammosphere-like structures, Tzbinduced reduction of JIMT1 MSFE was not statistically significant (Fig. 3B,C). These findings support further the notion that refractoriness to Tzb in HER2-positive BC cells can be explained in terms of $\mathrm{BC}$ stem/progenitor populations intrinsically resistant to Tzb. In this scenario, treatment with single-agent metformin was sufficient to drastically reduce MSFE in Tzb-refractory JIMT-1 cells ( $\sim 65 \%$ MSFE reduction at 100 $\mu \mathrm{mol} / \mathrm{L}$ metformin; Fig. $3 B, C)$. Perhaps more importantly, concurrent combination of Tzb and metformin worked synergistically to totally suppress the ability of JIMT-1 cells to form mammospheres. Thus, the ability of $1000 \mu \mathrm{mol} / \mathrm{L}$ metformin to decrease MSFE 
increased from about 5 times when used as single-agent to more than 22 times when combined with Tzb (Fig. 3C).

\section{Metformin interacts synergistically with Tzb to suppress the}

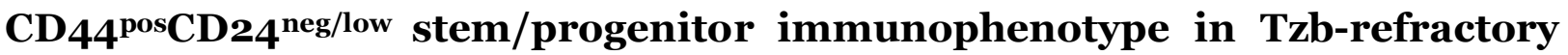
JIMT-1 BC cells. Given that Tzb-refractory HER2-overexpressing JIMT-1 cells constitutively express significant amounts of the transmembrane receptor CD44 [16] -a cardinal feature of BC stem cells- we sought to examine: 1.) Whether unsorted populations of JIMT-1 differentiated cultures were naturally enriched with the stem/progenitor CD44 $4^{\text {pos }} / \mathrm{CD} 24^{\text {neg/low }}$ progeny; and 2.) Whether concomitant exposure to $\mathrm{Tzb}$ and metformin significantly affected the $\mathrm{BC}$ stem $\mathrm{CD} 44^{\text {pos }} / \mathrm{CD} 24^{\text {neg/low }}$ immunophenotype. When using JIMT-1 cells from cultures passages \#20 to \#30, flow cytometry analyses clearly revealed a significant subpopulation of about 70\% of JIMT-1 cells naturally exhibiting the $\mathrm{CD} 44^{\text {pos }} / \mathrm{CD} 24^{\text {neg/low }} \mathrm{BC}$ stem profile (Fig. 3D). Rather than exhibiting a luminal-type classification, the unique distribution of CD24 \& CD44 markers in HER2-gene amplified JIMT-1 cells somewhat resembled that observed in

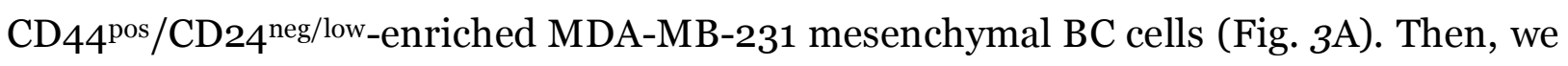
assessed distribution of the cell surface markers CD24 and CD44 in JIMT-1 cells following treatment $(48 \mathrm{~h})$ with two concentrations of metformin (i.e. 100 and 1000 $\mu \mathrm{mol} / \mathrm{L})$ in the absence or presence of an optimal dose $(100 \mu \mathrm{g} / \mathrm{mL})$ of Tzb (Fig. 3D). Whereas single-agent Tzb failed to significantly reduce the number of JIMT-1 cells exhibiting the putative stem-like CD44 ${ }^{\text {pos }} \mathrm{CD} 24^{\text {neg/low }}$ immunophenotype, $\mathrm{CD} 44^{\text {pos }} \mathrm{CD} 24^{\text {neg/low }}$ cell numbers were reduced by $\sim 30 \%$ upon treatment with 1000 $\mu \mathrm{g} / \mathrm{mL}$ metformin. Concurrent treatment with $\mathrm{Tzb}$ and $1000 \mu \mathrm{mol} / \mathrm{L}$ metformin reduced further, in a synergistic fashion, $\mathrm{CD} 44^{\mathrm{pos}} \mathrm{CD} 24^{\text {neg/low }}$ cell numbers (from $68 \%$ in untreated control cells to $37 \%$ in Tzb plus metformin co-treated cells) to concurrently 
increase the $\mathrm{CD} 44^{\text {pos }} \mathrm{CD} 24^{\text {pos }}$ fraction (from $32 \%$ in untreated control cells to $62 \%$ in Tzb plus metformin co-treated cells) (Fig. 3D).

To exclude that synergistic reduction of MSFE efficiencies in Tzb-refractory SKBR3 TzbR and JIMT-1 cells would simply reflect a generalized reduction in cellular viability, we replicated all the experimental groups in adherent conditions and cell growth was monitored by MTT assay. At the doses employed in sphere cultures, Tzb and metformin were ineffective at inhibiting cell growth of SKBR3 TzbR and JIMT-1 differentiated cultures routinely grown in plastic (data not shown). 


\section{DISCUSSION}

Hirsch et al. have recently demonstrated that tumor-forming, self-renewing BC stem cells appear to exhibit an exacerbated sensitivity to the anti-diabetic drug metformin [13]. In their hands, concurrent treatment with metformin (100 or $300 \mu \mathrm{mol} / \mathrm{L}$ ) and the cytotoxic doxorubicin was found to reduce tumor mass and prevent relapse much more effectively than either drug alone in a xenograft mouse model. Here, using three HER2-gene amplified $\mathrm{BC}$ in vitro models for Tzb-sensitivity (SKBR3), acquired Tzb auto-resistance (SKBR3 TzbR) and de novo Tzb refractoriness (JIMT-1), and employing mammosphere-forming efficiency \& mammosphere size as indicators of stem selfrenewal and progenitor cell proliferation, respectively, we provide for the first time preclinical evidence for the efficacy of the anti-diabetic drug metformin $(50-100 \mu \mathrm{mol} / \mathrm{L})$ to efficiently target Tzb-resistant tumor-initiating stem cells in HER2 overexpressors. We also confirm recent findings by Magnifico et al. showing that tumor-initiating cells of HER2-positive carcinoma cell lines are sensitive to the anti-HER2 monoclonal antibody Tzb [6]. Therefore, our findings corroborate and expand further Hirsch and Magnifico pioneer's findings to the combination of metformin with Tzb against HER2positive tumor-initiating $\mathrm{BC}$ stem cells.

First, we confirmed the distinctive ability of the anti-diabetic drug metformin to attack BC tumor-initiating stem cells within a heterogeneous population of BC cells overexpressing HER2. It should be noted that metformin concentrations as low as 50 $\mu \mathrm{mol} / \mathrm{L}$ were sufficient to mimic the therapeutic effects of Tzb against mammosphereinitiating cells in Tzb-naïve SKBR3 cells. Although the remarkable potency to eliminate stem/progenitor cells in sphere cultures of HER2 overexpressors became obvious when analyzing dose-dependent decreases in MSFE of SKBR3 sphere cultures supplemented with graded doses of metformin, it was relevant also that metformin caused gross 
morphological changes in mammosphere structure. It is reasonable to suggest that, during formation of multicellular "microtumors", metformin treatment may actively interfere with the basic processes of differentiation and/or apoptosis in the stem/progenitor cell population of Tzb-naïve, HER2-overexpressing BC cells.

Second, our current findings provide a novel scenario for the molecular understanding of Tzb resistance in HER2 gene-amplified BC (Fig. 4). The acquisition of Tzb resistance upon chronic exposure to Tzb can be explained in terms of emerging “Tzb-resistant” CD44-positive BC stem/progenitor cells because, in our hands, SKBR3 TzbR mammosphere-initiating cells did not respond to Tzb exposure. Our current findings strongly suggest that during acquisition of auto-resistance to Tzb (i.e. secondary resistance to Tzb), HER2-overexpressing luminal BC cells appear to undergo a transition to basal-like phenotypes characterized by a significant enrichment in cell populations overexpressing the stem-cell marker CD44. This CD44-related scenario for Tzb refractoriness was even more apparent in HER2-gene amplified BC cells with de novo resistance to multiple HER-targeted therapies. JIMT-1 BC cells not only exhibit primary resistance to Tzb but demonstrate further cross-resistance to several HER2inhibiting drugs including the HER-dimerization inhibitor antibody pertuzumab (2C4) and the small molecule tyrosine kinase inhibitors (TKIs) CI1033 (canertinib) and ZD1839 (gefitinib). Preliminary experiments in our laboratory demonstrated that JIMT-1 cells display also cross-resistance to the dual HER1/HER2 TKI lapatinib (data not shown). Given that Tzb treatment has been shown to fail at decreasing the fraction of JIMT-1 cells positive for ALDH1 [7], it has been suggested that BC stem cell-like phenotypes may underlie Tzb resistance in JIMT-1 cells [9]. In this regard, we have validated the notion that Tzb-refractory HER2-positive JIMT-1 cell cultures contain a significant population bearing the $\mathrm{CD} 44^{\text {pos }} \mathrm{CD} 24^{\text {neg/low }}$ phenotype initially described by $\mathrm{Al}-\mathrm{Hajj}$ et al. as a feature of $\mathrm{BC}$ stem cells that associates with the expression of 
basal/mesenchymal or myoepithelial markers [2]. Because earlier studies have repeatedly confirmed that all $\mathrm{BC}$ cell lines with significant $\mathrm{CD} 44^{\text {pos }} / \mathrm{CD} 24^{\text {neg/low }}$ subpopulation belong to the basal type of $\mathrm{BC}$ and display the phenotype of cancer cells that have undergone epithelial to mesenchymal transition (EMT) $[17,18]$, the unique distribution of CD24 and CD44 cell surface markers in HER2-overexpressing JIMT-1 cells may suggest that the induction of EMT in HER2-positive BC cells may generate cells with stem-like features, such as the $\mathrm{CD} 44^{\text {pos }} / \mathrm{CD} 24^{\text {neg/low }}$ phenotype and the ability to grow in the presence of HER2 inhibitors. In this regard, it is noteworthy that JIMT-1 cells failed to produce discrete and correctly organized spherical structures but rather formed somewhat loosely adhered clumps of cells when cultured in sphere medium for 7 days, a mammosphere-like architecture that has been observed in sphere cultures of

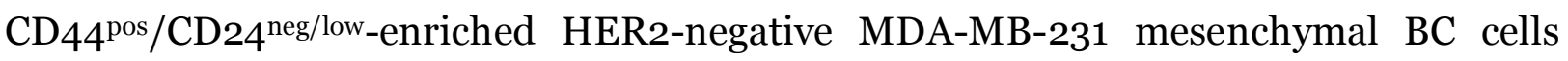
[10].

Third, mammosphere-initiating cells from both CD44-overexpressing SKBR3 TzbR cells with acquired auto-resistance to Tzb and CD44-overexpressing JIMT-1 cells with de novo resistance to Tzb retained an exquisite and somewhat exacerbated sensitivity to the inhibitory effects of metformin. It has previously been shown that mammosphere number reflects stem cell self-renewal, whereas mammosphere size reflects progenitor cell proliferation [11, 12]. Our current findings reveal that Tzb treatment may affect stem/progenitor cell proliferation in Tzb-refractory HER2-gene amplified BC because it significantly reduced mammosphere size. The incapability of Tzb to significantly decrease mammosphere cell number strongly suggests that Tzb efficacy in HER2overexpressors rather relates to its ability to block HER2-driven stem/progenitor cell self-renewal. Of note, metformin co-treatment synergistically worked with Tzb to suppress self-renewal and proliferation of Tzb-refractory tumor-initiating cells In this scenario, it would be tempting to suggest that metformin-targeted crucial survival 
mechanisms may underlie also Tzb resistance in tumor-initiating cells because concurrent combination of metformin with Tzb synergistically reduced MSFE as well as mammosphere size in Tzb-refractory SKBR3 TzbR and JIMT-1 cells. Given that the percentage of $\mathrm{CD} 44^{\text {pos }} \mathrm{CD} 24^{\text {neg/low }}$ tumor-initiating cells has been shown to significantly correlate with MSFE in BC [5] and that co-treatment with metformin and Tzb synergistically reduced the percentage of JIMT-1 cells exhibiting the $\mathrm{CD} 44^{\text {pos }} \mathrm{CD} 24^{\text {neg/low }}$ stem/progenitor immunophenotype, it would be relevant to elucidate whether, in the presence of metformin, “Tzb-resistant” BC stem/progenitor cells may regain sensitivity to Tzb.

In summary, we present the first evidence that the anti-diabetic drug metformin synergistically interacts with molecularly targeted agents such as Tzb to suppress selfrenewal (measured as MSFE) and proliferation (evaluated as mammosphere size) of tumor-initiating (BC stem) cells of HER2-positive carcinoma cells showing acquired and de novo resistance to Tzb. The therapeutic plasma levels of metformin in clinical medicine are 0.465 to $2.5 \mathrm{mg} / \mathrm{mL}$, whereas we observed statistically significant reductions in MSFE and mammosphere size in 50 to $1000 \mu \mathrm{g} / \mathrm{mL}$ metformin, a minimum 3-fold excess over the recommended therapeutic levels. Although the range of concentrations employed in our current approach were significantly lower than those used in earlier studies exposing cultures of differentiated cancer cells to metformin concentrations ranging from 1 to $100 \mathrm{mmol} / \mathrm{L}$, forthcoming studies should validate whether concentrations of metformin capable of efficiently eliminating mammosphereinitiating cells might be also attained during breast cancer treatment. Although it has been reported that metformin can accumulate in tissues at concentrations several-fold higher than those in blood [19], caution must be therefore applied when extrapolating our current in vitro results into clinical practice. Nevertheless, it is reasonable to suggest that, in combination with conventional chemotherapy and HER2-targeting 
agents, metformin co-treatment may add an important therapeutic benefit in debulking and in targeting tumor-initiating cells of HER2-overexpressing BC [20, 21]. Although it remains to be elucidated which metformin-sensitive molecular mechanisms control the maintenance and survival of Tzb-resistance HER2-gene amplified tumor initiating cells, crucial evidence of the clinical efficacy of MET could be obtained from "proof-ofprinciple" studies with neoadjuvant regimens including neoadjuvant chemotherapy (to target bulk BC cells) and Tzb with MET (both targeting self-renewal and proliferation of tumor-initiating cells) in women diagnosed with HER2-positive primary BC [22].

Conflicts of Interest: None to declare

\section{Acknowledgments}

Alejandro Vazquez-Martin is the recipient of a "Sara Borrell" post-doctoral contract (CDo8/o0283, Ministerio de Sanidad y Consumo, Fondo de Investigación Sanitaria FIS-, Spain). Work at Menendez' Laboratory is supported by Instituto de Salud Carlos III (Ministerio de Sanidad y Consumo, Fondo de Investigación Sanitaria -FIS-, Spain, Grants CPo5-0009o and PIo6-0778 and RDo6-0020-0028), the Fundación Científica de la Asociación Española Contra el Cáncer (AECC, Spain), and by the Ministerio de Ciencia e Innovación (SAF2009-11579, Plan Nacional de I+D+ I, MICINN, Spain). 


\section{REFERENCES}

1. Jones RJ, Matsui WH, Smith BD: Cancer stem cells: are we missing the target? J Natl Cancer Inst 96:583-585, 2004.

2. Al-Hajj M, Wicha MS, Benito-Hernandez A, Morrison SJ, Clarke MF: Prospective identification of tumorigenic breast cancer cells. Proc Natl Acad Sci USA 100:39833988, 2003.

3. Fillmore CM, Kuperwasser C: Human breast cancer cell lines contain stem-like cells that self-renew, give rise to phenotypically diverse progeny and survive chemotherapy. Breast Cancer Res 10:R25, 2008.

4. Kakarala M, Wicha MS: Implications of the cancer stem-cell hypothesis for breast cancer prevention and therapy. J Clin Oncol 26:2813-2820, 2008.

5. Li X, Lewis MT, Huang J, Gutierrez C, Osborne CK, Wu MF, Hilsenbeck SG, Pavlick A, Zhang X, Chamness GC, Wong H, Rosen J, Chang JC: Intrinsic resistance of tumorigenic breast cancer cells to chemotherapy. J Natl Cancer Inst 100:672-679, 2008.

6. Magnifico A, Albano L, Campaner S, Delia D, Castiglioni F, Gasparini P, Sozzi G, Fontanella E, Menard S, Tagliabue E: Tumor-initiating cells of HER2-positive carcinoma cell lines express the highest oncoprotein levels and are sensitive to trastuzumab. Clin Cancer Res 15:2010-2021, 2009.

7. Korkaya H, Paulson A, Iovino F, Wicha MS: HER2 regulates the mammary stem/progenitor cell population driving tumorigenesis and invasion. Oncogene 27:6120-6130, 2008.

8. Ginestier C, Hur MH, Charafe-Jauffret E, Monville F, Dutcher J, Brown M, Jacquemier J, Viens P, Kleer CG, Liu S, Schott A, Hayes D, Birnbaum D, Wicha MS, 
Dontu G: ALDH1 is a marker of normal and malignant human mammary stem cells and a predictor of poor clinical outcome. Cell Stem Cell 1:555-567, 2007.

9. Bedard PL, Cardoso F, Piccart-Gebhart MJ: Stemming resistance to HER-2 targeted therapy. J Mammary Gland Biol Neoplasia 14:55-66, 2009.

10. Grimshaw MJ, Cooper L, Papazisis K, Coleman JA, Bohnenkamp HR, ChiaperoStanke L, Taylor-Papadimitriou J, Burchell JM: Mammosphere culture of metastatic breast cancer cells enriches for tumorigenic breast cancer cells. Breast Cancer Res 10:R52, 2008

11. Dontu G, Abdallah WM, Foley JM, Jackson KW, Clarke MF, Kawamura MJ, Wicha MS: In vitro propagation and transcriptional profiling of human mammary stem/progenitor cells. Genes Dev 17:1253-1270, 2003.

12. Dontu G, Jackson KW, McNicholas E, Kawamura MJ, Abdallah WM, Wicha MS: Role of Notch signaling in cell-fate determination of human mammary stem/progenitor cells. Breast Cancer Res 6:605-615, 2004.

13. Hirsch HA, Iliopoulos D, Tsichlis PN, Struhl K: Metformin selectively targets cancer stem cells, and acts together with chemotherapy to block tumor growth and prolong remission. Cancer Res 69:7507-7511, 2009.

14. Vazquez-Martin A, Oliveras-Ferraros C, Menendez JA: Autophagy facilitates the development of breast cancer resistance to the anti-HER2 monoclonal antibody trastuzumab. PLoS One 4:e6251, 2009.

15. Tanner M, Kapanen AI, Junttila T, Raheem O, Grenman S, Elo J, Elenius K, Isola J: Characterization of a novel cell line established from a patient with Herceptin-resistant breast cancer. Mol Cancer Ther 3: 1585-1592, 2004. 
16. Pályi-Krekk Z, Barok M, Isola J, Tammi M, Szöllosi J, Nagy P: Hyaluronan-induced masking of ErbB2 and CD44-enhanced trastuzumab internalisation in trastuzumab resistant breast cancer. Eur J Cancer 43:2423-2433, 2007.

17. Sheridan C, Kishimoto H, Fuchs RK, Mehrotra S, Bhat-Nakshatri P, Turner CH, Goulet R Jr, Badve S, Nakshatri H: CD44+/CD24- breast cancer cells exhibit enhanced invasive properties: an early step necessary for metastasis. Breast Cancer Res 8:R59, 2006.

18. Honeth G, Bendahl PO, Ringnér M, Saal LH, Gruvberger-Saal SK, Lövgren K, Grabau D, Fernö M, Borg A, Hegardt C: The CD44+/CD24- phenotype is enriched in basal-like breast tumors. Breast Cancer Res 10:R53, 2008.

19. Wilcock $\mathrm{C}$ and Bailey CJ: Accumulation of metformin by tissues of the normal and diabetic mouse. 24:49-57, 1994.

20. Gonzalez-Angulo AM, Meric-Bernstam F. Metformin: A Therapeutic Opportunity in Breast Cancer. Clin Cancer Res 2010 Mar 9. [Epub ahead of print]

21. Martin-Castillo B, Vazquez-Martin A, Oliveras-Ferraros C, Menendez JA: Metformin and cancer: Doses, mechanisms and the dandelion and hormetic phenomena. Cell Cycle 9: 1057-1064, 2010.

22. Martin-Castillo B, Dorca J, Vazquez-Martin A, Oliveras-Ferraros C, Lopez-Bonet E, Garcia M, Del Barco S, Menendez JA: Incorporating the antidiabetic drug metformin in HER2-positive breast cancer treated with neo-adjuvant chemotherapy and trastuzumab: an ongoing clinical-translational research experience at the Catalan Institute of Oncology. Ann Oncol 21:187-189, 2010. 


\section{FIGURE LEGENDS}

\section{Figure 1. Metformin is as efficient as Tzb at inhibiting mammosphere}

formation in Tzb-naïve HER2-overexpressing SKBR3 cells. A. Figure shows representative light microscope representations of mammospheres formed by SKBR3 cells growing in sphere medium for 7 days in the absence or presence of $10 \mu \mathrm{g} / \mathrm{mL} \mathrm{Tzb}$ and co-exposed to increasing concentrations of metformin, as specified (10x magnifications). B. MSFE of SKBR3 cells was calculated as the number of mammospheres (diameter $>50 \mu \mathrm{m}$ ) formed in 7 days divided by the original number of cells seeded and expressed as percentage means (columns) \pm SD (bars). Re-feeding of mammospheres cultures with experimental agents and/or sphere medium was performed on days 3 and $5 . \mathrm{P}<0.01$ and $\mathrm{P}<0.001$, statistically significant differences from the control group. N. S. Not statistically significant. C. Fold-decreases in the MSFE of SKBR3 cells growing in sphere medium supplemented with Tzb in the absence or presence of graded concentrations of metformin were calculated by dividing MSFE in untreated control sphere cultures by those obtained in the presence of Tzb and/or metformin. Tzb: Trastuzumab; MET: Metformin; Scale bars: $100 \mu$.

Figure 2. Metformin and Tzb interact synergistically to suppress selfrenewal and proliferation of tumor-initiating cells in Tzb-conditioned SKBR3 TzbR cells. A. Presented are representative flow cytometry dot plots for the expression of CD44 and CD24 cell markers in Tzb-naïve SKBR3 parental cell line and its derivative Tzb-resistant SKBR3 TzbR cells (insert: CD44 and CD24 distribution in basal-like MDA-MB-468). The results of this analysis are summarized with respect to four cell population fractions: $\mathrm{CD} 44^{\text {pos }} \mathrm{CD} 24^{\text {neg }}, \mathrm{CD} 44^{\text {neg }} \mathrm{CD} 24^{\text {pos }}, \mathrm{CD} 44^{\text {pos }} \mathrm{CD} 24^{\text {pos }}$, and $\mathrm{CD} 44^{\text {neg }} \mathrm{CD} 24^{\text {neg. }}$. Figure shows also representative light microscope-high 
magnifications (20x) of mammospheres structures formed by SKBR3 and SKBR3 TzbR cells growing in sphere medium for 7 days. B. Figure shows representative light microscope representations of mammospheres-like structures formed by SKBR3 TzbR cells growing in sphere medium for 7 days in the absence or presence of $100 \mu \mathrm{g} / \mathrm{mL} \mathrm{Tzb}$ and co-exposed to increasing concentrations of metformin, as specified (10x magnification). C, D. SKBR3 TzbR MSFE and fold-decreases of MSFE observed in SKBR3 TzbR cells growing in sphere medium supplemented with Tzb in the absence or presence of graded concentrations of metformin were calculated as in Fig. 1B,C. Tzb: Trastuzumab; MET: Metformin; Scale bars: $100 \mu \mathrm{m}$.

Figure 3. Metformin and Tzb interact synergistically to suppress selfrenewal and proliferation of tumor-initiating cells in JIMT-1 cells with resistance to HER2-targeted therapies ab initio. A. Presented are representative flow cytometry dot plots for the expression of CD44 and CD24 cell markers in Tzb-refractory HER2-overexpressing JIMT-1 BC cells and in MDA-MB-231 mesenchymal BC cells. The results of this analysis are summarized as in Fig. $2 A$. Figure shows also representative light microscope-high magnifications (20x) of mammospheres-like structures formed by JIMT-1 cells growing in sphere medium for 7 days. B. Figure shows representative light microscope representations of mammospheres-like structures formed by JIMT-1 cells growing in sphere medium for 7 days in the absence or presence of $100 \mu \mathrm{g} / \mathrm{mL}$ Tzb and co-exposed to increasing concentrations of metformin, as specified (10x magnifications). C. JIMT-1 MSFE and fold-decreases of MSFE observed in JIMT-1 cells growing in sphere medium supplemented with $\mathrm{Tzb}$ in the absence or presence of graded concentrations of metformin were calculated as in Fig. $1 B, C$. D. Presented are representative flow 
cytometry dot plots for the expression of CD44 and CD24 cell markers in JIMT-1 cells treated or not with Tzb, metformin, and Tzb plus metformin, as specified. The results of this analysis are summarized as in Fig. $2 A$. Identification of the $\mathrm{CD} 44^{\text {pos }} \mathrm{CD} 24^{\text {neg/low }}$ stem/progenitor sub-population is highlighted (dashed red line). Tzb: Trastuzumab; MET: Metformin; Scale bars: $100 \mu \mathrm{m}$.

Figure 4. Metformin and Tzb-resistant tumor-initiating BC stem cells: A working model. The efficacy of HER2-targeted therapies such as Tzb may be explained by their direct activity against HER2-positive BC stem cells [6, 7, 9]. Conversely, BC stem cells may be also at the root of resistance to Tzb. On the one hand, molecular characteristics of HER2-positive BC stem cells (e.g. expression levels of the stem cell marker CD44) may evolve under the selection pressure of Tzb, driving the selection and/or emergence of a sub-population of HER2-positive BC stem cells with acquired resistance to Tzb. On the other hand, the induction of the epithelialmesenchymal transition (EMT) cellular program (prior or during Tzb challenge) may generate HER2-positive cells with stem-like features, such as the Tzb-refractory $\mathrm{CD} 44^{\text {pos }} \mathrm{CD} 24^{\text {neg/low }}$ phenotype. Pro-survival mediators of $\mathrm{BC}$ stem cell resistance to $\mathrm{Tzb}$ appear to be exquisitely sensitive to the anti-diabetic drug metformin thus eliciting additive and supra-additive inhibitory interactions between metformin and Tzb against HER2-positive BC stem cells. Beyond providing an explanation for Tzb treatment failure, our current findings provide a strong rationale for studying the clinical relevance of yet to be defined metformin-targeted BC stem-cell maintenance pathways in combination with Tzb. 
A

[MET]
- Tzb

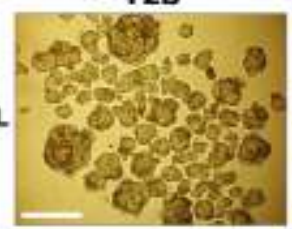

$0 \mu \mathrm{mol} / \mathrm{L}$

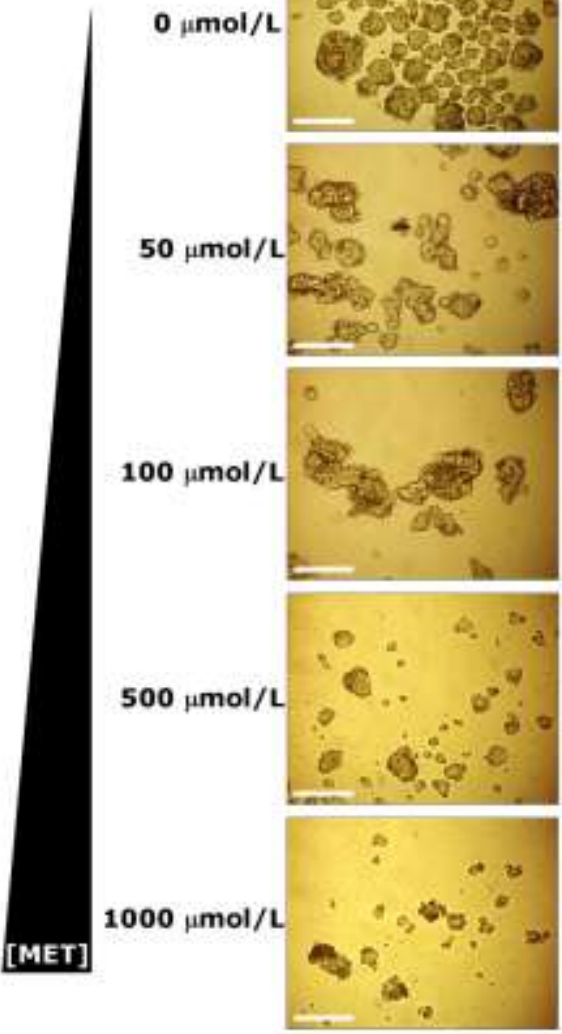

$+10 \mu \mathrm{g} / \mathrm{mL}$ Tzb
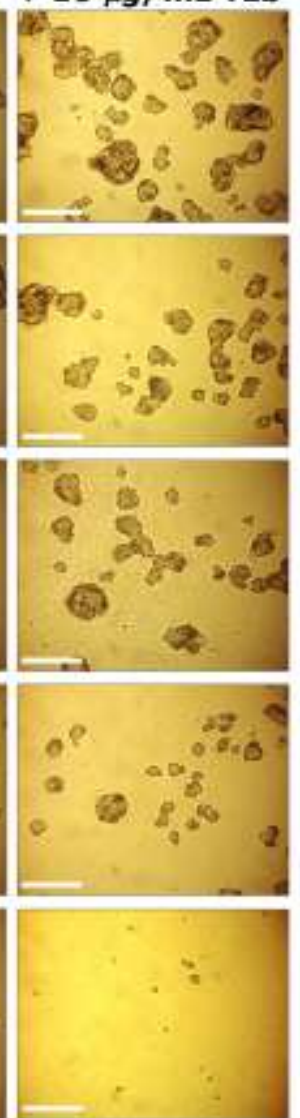

B

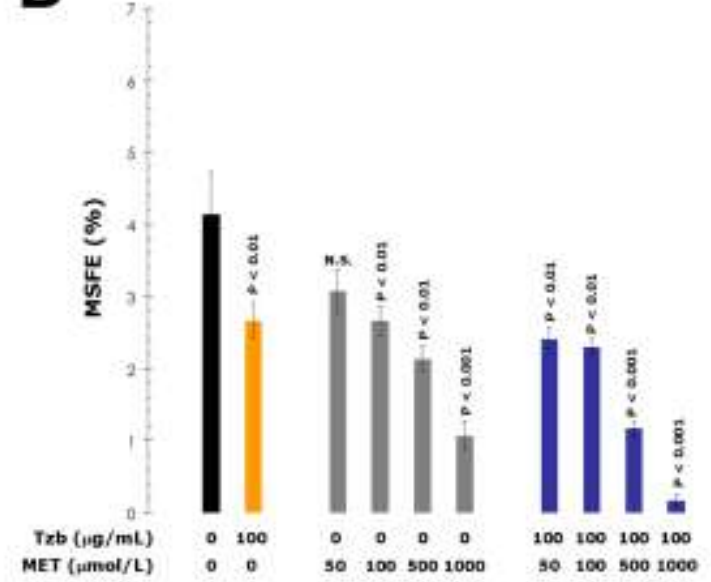

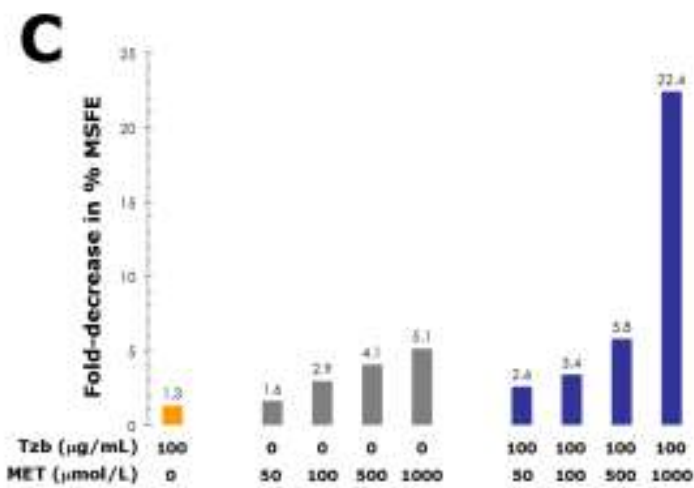


A

SKBR3

SKBR3 TzbR
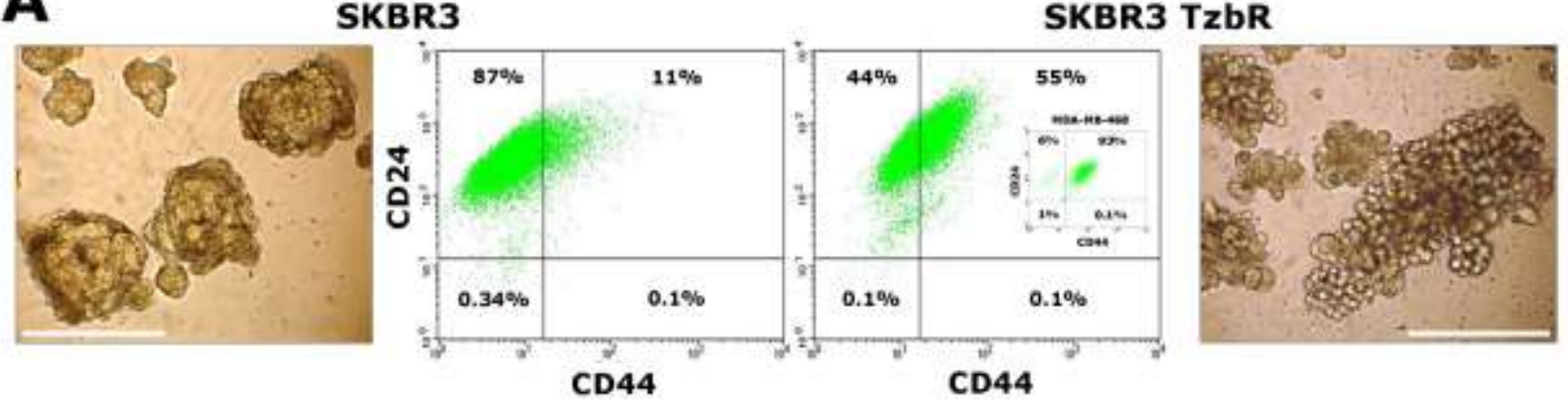

B

- Tzb $\quad+100 \mu g / m L ~ T z b$

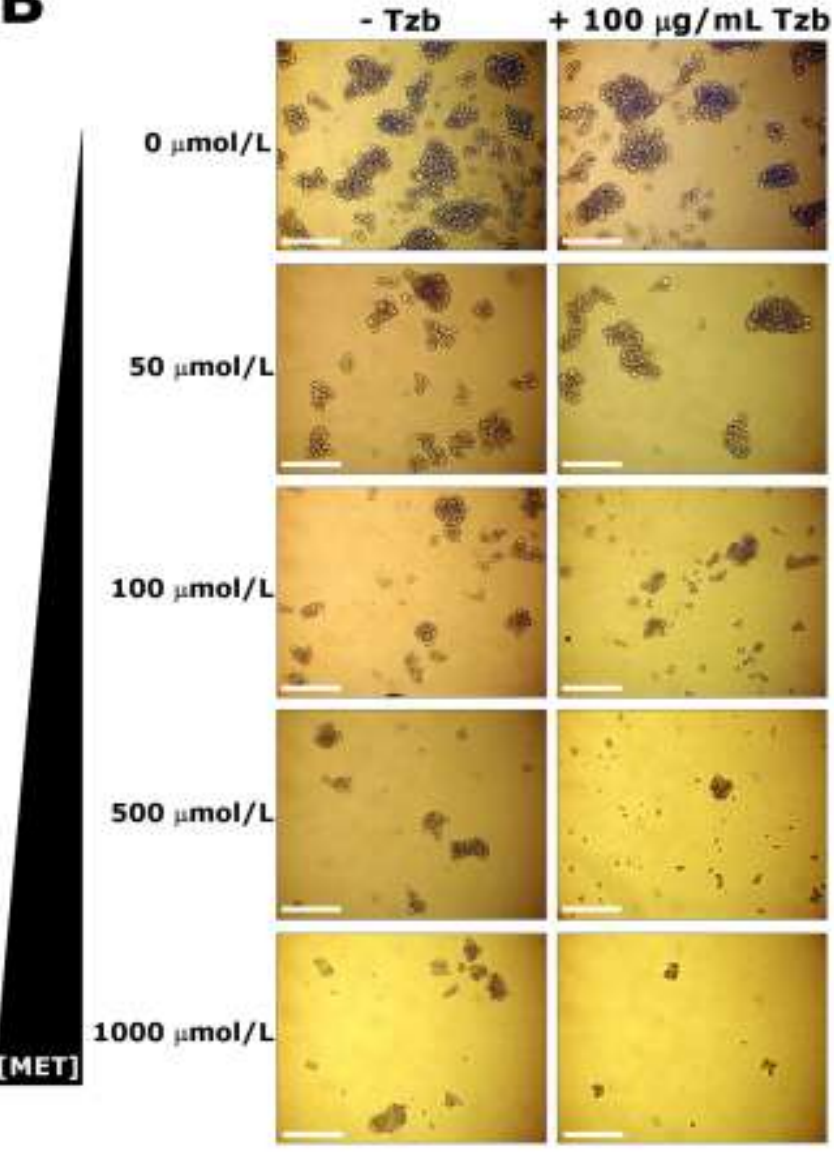

C
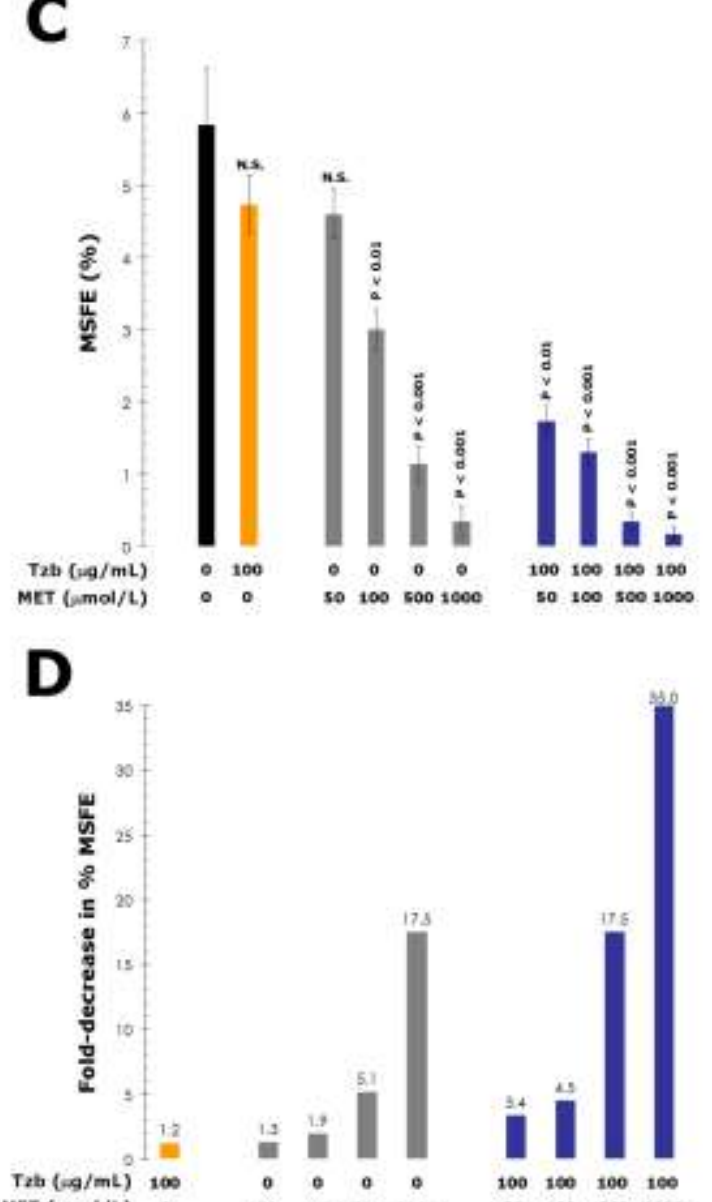

MET (umal/L) o
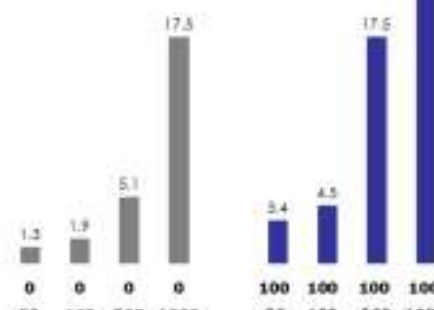

$100 \quad 100 \quad 100 \quad 100$ s0 $100 \quad 5001000$ 


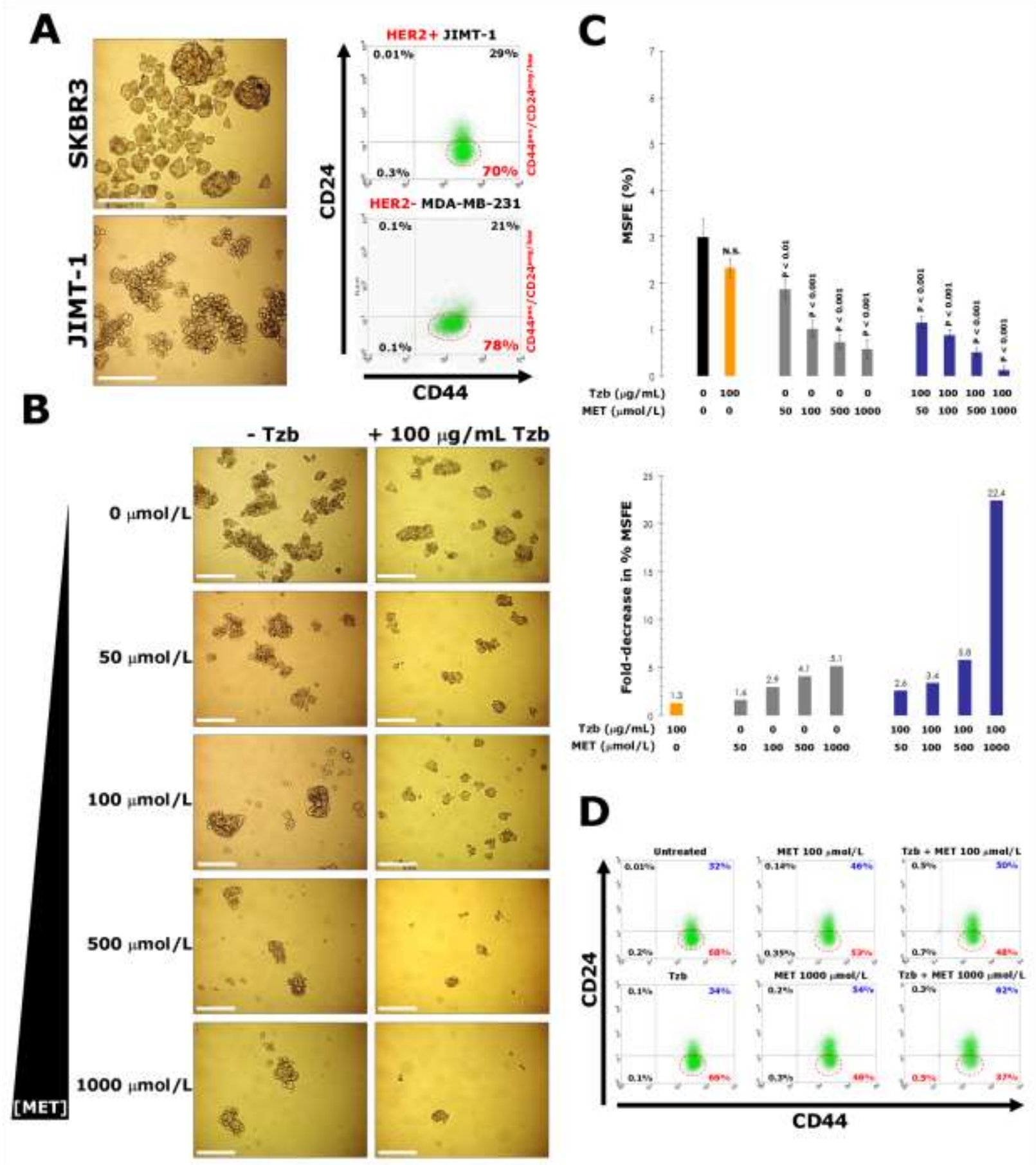




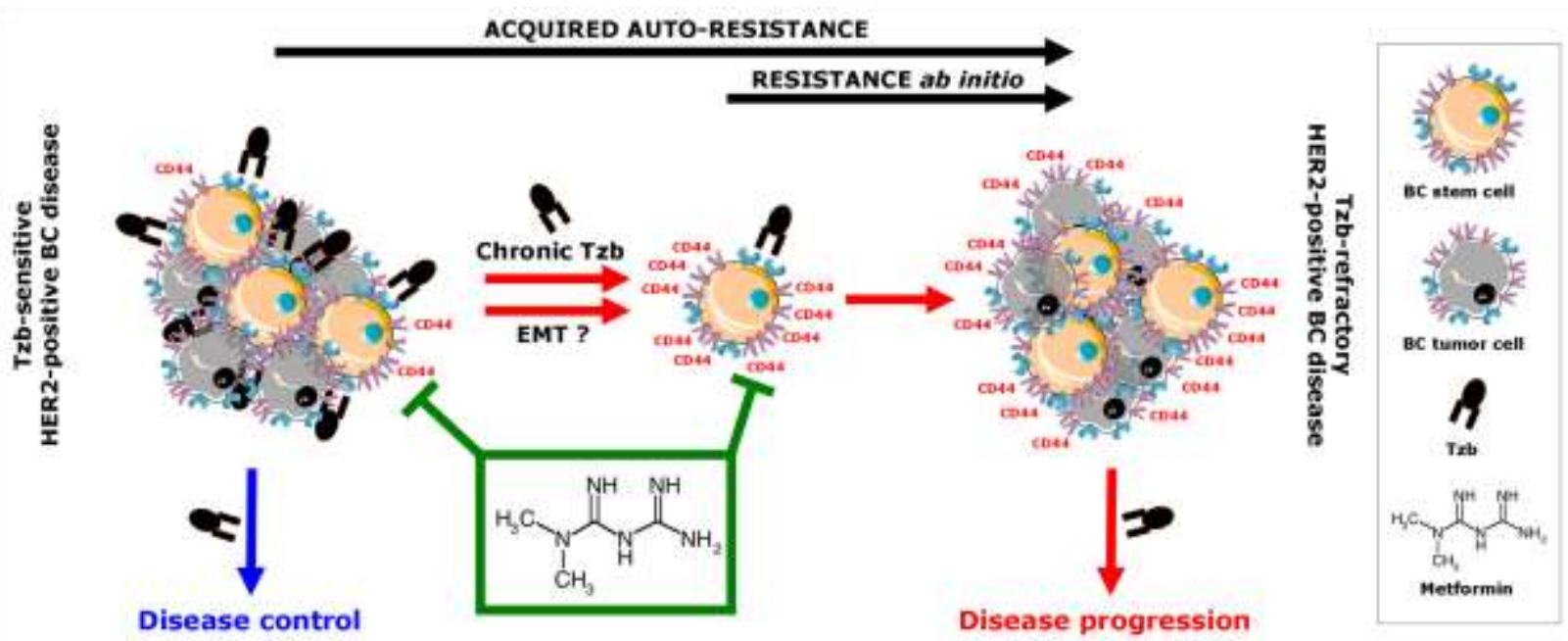

